\title{
IRRATIONAL RESISTANCE OR IRRATIONAL SUPPORT? PERFORMANCE EFFECTS OF PROJECT LEADER STATUS
}

\author{
BALAZS SZATMARI \\ Erasmus University \\ Burgemeester Oudlaan 50 \\ 3062 PA Rotterdam, the Netherlands \\ DIRK DEICHMANN \\ Erasmus University \\ JAN VAN DEN ENDE \\ Erasmus University
}

\begin{abstract}
In this study, we explore the effects of project leader status in new product development projects. We predict and find that that project leader status increases project performance up to a certain point after which it decreases performance. Further, status increases the variability of project performance, that is, it leads to more extreme performance in both directions.
\end{abstract}

\section{INTRODUCTION}

Developing new products and services plays a critical role in creating higher organizational performance and greater competitive advantage. However, it is, at the same time, a highly uncertain endeavor. Vigorously pursuing certain new product development projects may lead to great successes but also to great failures (Royer, 2003). In fact, "bankruptcy courts are full of companies that should have been less zealous in pursuing some [new product development] projects" (Schilling, 2009: 244). Why do some firms select and remain zealous in sustaining failing projects?

We argue that project leaders with a high status can be both beneficial and detrimental to organizations. While status is a valuable asset up to a certain point that opens up opportunities inaccessible to others (Bothner, Kim, \& Smith, 2011), high-status project leaders are also motivated to exploit their influence and network to keep their projects alive even when the performance expectations are not so promising. As such, we expect that status has an inverted Ushaped relationship with project performance, and increases performance extremeness (i.e., the variability of project performance). In order to test our hypotheses, we analyzed social network data retrieved from an extensive online database of videogames.

Traditionally, the literature on social networks has associated social capital with higher performance (e.g., Moran, 2005; Rodan \& Galunic, 2004). Moreover, researchers of new product development processes have generally argued that the exploitation of status and networks leads to increased performance (Ancona \& Caldwell, 1992; Sethi, Iqbal, \& Sethi, 2012). We contribute to both streams of literature by showing how status, measured through a person's social capital (Bonacich, 1987), is associated with performance, but also with performance extremeness (Chatterjee \& Hambrick, 2007; Sanders \& Hambrick, 2007). This means that high status can lead both to very successful projects but also to projects that fail on the market. It is important to learn 
more about the antecedents of failures, as often a single failure is enough to endanger the livelihood of organizations (e.g., Schilling, 2009).

\section{THEORETICAL BACKGROUND AND HYPOTHESES}

Product development processes are inherently risky, as the associated development costs are high and the probability of product success is low (Schilling, 2009). Hence, with the objective of selecting the development projects that can provide the highest revenues and incur the lowest cost, organizations often apply a formal selection and review process (Barczak, Griffin, \& Kahn, 2009). However, previous research has shown that project acceptance and sustainment cannot be entirely explained by these factors (Green, Welsh, \& Dehler, 2003). For instance, Sleesman, Conlon, McNamara, and Miles (2012) conducted a meta-analysis in which they found a large number of factors that contribute to the sustainment of commitment to failing projects. Thus, it can be concluded that new product development projects are particularly exposed to decision errors due to the high level of uncertainty they incorporate (e.g., Moenaert \& Souder, 1990; Shenhar \& Dvir, 1996). Therefore, it can be expected that informal mechanisms play a more significant role in new product development decisions than in less uncertain contexts.

The effect of status, as an indicator of high-quality activities, has been highly neglected in the new product development literature. This is surprising given the fact that status is more important under uncertain conditions (Podolny, 1994). Arguably, product development projects are very uncertain (Moenaert \& Souder, 1990). An exception to the dearth of research about the role of status in new product development projects is the recent study of Reitzig and Sorenson (2013). The authors found that middle-managers are negatively biased toward the acceptance of project ideas from subunits different than their own. However, this negative biasing effect is mitigated by the status of the subunit from which the project idea originates. We further extend these the insights by exploring the market consequences (i.e., performance and performance extremeness) of the effect of status.

\section{Status and Performance}

Previous research has already shown evidence of a positive relationship between status and performance (e.g., Stuart, Hoang, \& Hybels, 1999). High status of individuals usually implies a more central network position (Bonacich, 1972; Oldroyd \& Morris, 2012). A central network position, in turn, has been associated with stronger influence (Brass \& Burkhardt, 1993) and higher product development performance (e.g., Burt, 2004; Durmuşoğlu, 2013). Thus, a project leader's status, as indicated by his or her central network position, should have a positive influence on project performance. This is because a higher status of a project leader provides him or her with access to high quality resources (e.g., Ancona and Caldwell, 1992). Moreover, since high-status individuals are more powerful than others, there is less need for them to make compromises with other organizational members that could adversely affect the new product's fit with the market in order to implement their projects (Sethi et al., 2012).

However, a very high status can also have negative consequences. Bothner et al. (2012) argue that gaining status is an end in itself. Hence, high status might lead to complacency (i.e., lack of motivation to perform even better). Furthermore, due to their network position, highstatus project leaders are often sought for help and advice, because they are highly visible, and 
perceived to be highly competent (Oldroyd \& Morris, 2012). This likely leads to distraction and information-overload. Both of these effects, in turn, should have a negative impact on performance. Therefore, we predict that very high status may decrease performance of project leaders.

Hypothesis 1. There is an inverted "U-shaped" relationship between project leader status and project performance.

\section{Status and Performance Extremeness}

Centrally located individuals, such as high-status project leaders, are in a particularly advantageous position to convince others in the organization about their projects (Burt, 1992; Royer, 2003). Moreover, if project reviewers have any doubt with respect to the project itself, these concerns are more likely to disappear when a high-status project leader attempts to dispel them because of high-status organizational members`competent image (Oldroyd \& Morris, 2012). Due to the high level of uncertainty that surrounds new product development projects, reviewers cannot rely on accurate quantitative expectations. In such a case, project leader status might be a strong factor that influences reviewers' decisions. Finally, since high-status individuals usually want to protect their position in the organization (Ridgeway \& Walker, 1995), they might do what they can to overcome any organizational resistance. Hence, status can not only suppress irrational resistance but can also generate irrational support. Therefore, we propose that high-status project leaders, due to their network position and image, are in a better position to launch projects on the market that will deviate from the expected performance not only in the very positive, but also the very negative direction.

Hypothesis 2. The project leader's status is positively associated with the extremeness of project performance.

\section{SETTING AND METHOD}

We tested our hypotheses with data retrieved from MobyGames.com, an extensive online database that contains information about most of the videogames developed from 1972 until today. We gathered longitudinal social network data of 12 companies, which were consistently holding about $60 \%$ of the global market share between 2008 and 2012. For this sample, we looked at the status of main game producer who can be considered as the project leader in this type of industry (Irish, 2005). Our sample consists of 745 projects of 468 game producers. We built mixed-effects models because of within-cluster dependence. We assumed that performance follows a normal, whereas performance extremeness follows a skewed distribution. Therefore, we applied linear and generalized linear mixed-effects models to predict our dependent variables. Before entering the variables in the models, we mean-centered all continuous variables to reduce multicollinearity (except for performance extremeness to avoid negative values). Finally, we multiplied status scores by 100 for the ease of interpretation.

\section{Dependent Variables}

Project performance. We measure project performance by taking the mean of the standardized values of the average user rating and critique review scores that can be found on the website MobyGames.com. 
Performance extremeness. To operationalize this construct, we first regressed performance on all our variables, including status and status squared, to predict performance that can be reasonably expected from project leaders $\left(\chi^{2}=6964.9, p<.0001\right)$. In order to capture the deviation from predicted performance, we took the absolute value of the residuals of this regression, as we were interested in the absolute deviation, regardless of its direction (Sanders \& Hambrick, 2007).

\section{Independent Variable}

Status. Status is measured by applying Bonacich's centrality measure (Bonacich, 1987; Podolny, 2005):

$$
S_{i k t}(\alpha, \beta)=\sum_{j}\left(\alpha+\beta S_{j k t}\right) d_{i j t}
$$

where $S_{i k t}$ denotes the status of actor $i$, in firm $k$, at time $t$, where $t$ is the release year of the game in question, and $\mathrm{d}_{\mathrm{ijt}}$ denotes whether nodes $\mathrm{i}$ and $\mathrm{j}$ have launched a project together in the previous three years before time t. $\beta$ is set to $3 / 4$ of the inverse of the highest normed eigenvalue of the corresponding affiliation matrix, following prior network analytic studies (Bothner et al., 2011; Podolny, 2005). Moreover, we rescaled the centrality scores, such that they sum up to one for each year in each firm.

\section{Control Variables}

Project level variables. We include the year of release of the game as a continuous variable to control for trends, technological changes, and changing customer and critique perceptions over time. We also control for both newness-to-market and newness-to-firm of the project as an indicator of risk and organizational resistance associated with a project, and the producer`s ability to flexibly interpret the new product (Sethi et al., 2012). To get these variables, we used 62 category codes based on information that can be found on the website we analyzed. The category codes can take values 0 or 1, 1 meaning that the game fits in a certain category (e.g., action game, racing game, etc.). Newness-to-market is included in our models as a dummy variable, where 1 means that the combination of category codes of the project is completely new. That is to say, there had been no similar game published before the year when the game in question was published (in time t). Newness-to-firm is calculated in a similar way, but the project's category codes are compared only to those of other projects developed or published by only the company in question before time t. Further, we include the project's target market, since it may affect performance and the project leader's ability to sell the project to top-management or project reviewers, because each target market might be associated with different levels of risk. The target market is determined by the scores of the Entertainment Software Rating Board (ESRB). ESRB assigns age and content ratings for video games and specifies the appropriate target group for each videogame. It may assign the following ratings: everyone, everyone+10, kids to adults, mature, and teen. We control for target market by including four dummy variables with "everyone" being the reference category. Moreover, whether a game is licensed or not might affect performance and other organizational members' perceptions outside the boundaries of the project. A licensed game is an adaptation of a movie, a comic book, or a TV show. Since such videogames leverage a lot of attention from these other media, developer companies might 
allocate less resource to such projects as a lower quality product also can ensure an adequate amount of revenues. We control for this effect by including a dummy variable, 1 meaning that the game is licensed. We also control for whether a project is externally developed, that is, the publisher company is not identical to the developer company. We do so because an externally developed project might get a different amount of resource compared to internally developed ones, there is a higher level of division of labor in the development and publishing activities, and it might affect a producer's ability to create commitment to the project. Finally, we also include team size as a proxy for the development budget.

Producer level variables. We control for the presence of structural holes, because it might affect an individual's innovation-related performance and his or her ability to broker between alters (Burt, 1992; 2004). To compute structural holes, we first applied Burt`s constraint measurement which is given by the following formula (Burt, 2004):

$$
c_{i j}=\left[p_{i j}+\sum_{q} p_{i q} p_{q j}\right]^{2} ; i \neq j
$$

where $p_{i j}$ is the proportion of $i$ s network time and energy invested in actor $j$. We then computed $1-\sum_{i} c_{i j}$ for each actor. We also control for the number of parallel projects a producer implements in year $t$, since this might significantly affect his or her ability to allocate enough time to the management of the project in question. In addition, we control for the project leader's experience, which is the sum of all projects the producer implemented in the previous years.

Firm level variables. It is important to control for the number of firm projects implemented by the firm in question in $\mathrm{t}-1, \mathrm{t}-2$, and $\mathrm{t}-3$, because a large number of implemented projects, might be an indication of a generally low resistance against novel projects and a flexible project review process. Further, we also included firm innovativeness, because novel projects might face less resistance in firms, which are more open to highly novel projects (Sethi et al., 2012). To construct firm innovativeness, we aggregated the number of projects which were newto-market in a firm's project portfolio in year $\mathrm{t}-1, \mathrm{t}-2$ and $\mathrm{t}-3$, and divided it by the sum of all firm projects implemented in the same time window to determine firm innovativeness in a given year. We applied a moving time window for firm level variables, because firm innovativeness, hence the company culture and the review process, might change over time.

\section{RESULTS}

Hypothesis 1 predicted that there is an inverted U-shaped relationship between status and project performance. Our results confirm Hypothesis 1, as we find that the coefficient of the linear term of status is positive $(\beta=11.64, p<.05)$, and that of the squared term of status is significant and negative $(\beta=-7.11, p<.05)$. We also find support for Hypothesis 2 which predicted a positive linear association between status and performance extremeness $(\beta=0.58, p$ $<.01)$. Thus, the positive effect of status on project performance turns to be negative for very high values of status, while status has a linear positive effect on performance extremeness. This implies that project leaders with a very high status not only exhibit decreasing performance, but also high variation in performance, in both directions.

\section{DISCUSSION}


In this study, we show how project leader status affects project performance and performance extremeness. In line with our theory, we find that project leader status has an inverted U-shaped effect on project performance and a positive effect on performance extremeness. This suggests that project leaders with a very high status are more often associated with failed projects compared with project leaders who have a medium status level, presumably because of their ability to gather support for valuable projects, but also for less valuable ones.

Whereas scholars often argue that social capital is beneficial in a product development context (e.g., Moran, 2005), we show that status derived from social capital might have detrimental effects for new product development. We also shed more light on the effects of networking and coalition building in product development. It has been argued that teams and idea owners can benefit from political tactics and networking, as it may result in higher idea acceptance rate (Baer, 2012), access to high-quality resources (Ancona \& Caldwell, 1992), and higher market performance (Sethi et al., 2012). Contrary, we find that high-status project leaders are often associated with low-performing projects. Finally, we offer important contributions to both of the aforementioned research streams by demonstrating that high status not only leads to decreasing performance, but also to extreme performance. As such, status is not only associated with great successes, but also with great failures. Previous research has paid increasing attention to performance extremeness. We show that next to narcissism (Chatterjee \& Hambrick, 2007) and a compensation system (Sanders \& Hambrick, 2007), project leader status is an important antecendent of extreme performance.

\section{REFERENCES AVAILABLE FROM THE AUTHORS}

